\title{
Human umbilical cord blood cells rescued traumatic brain injury- induced cardiac and neurological deficits
}

\author{
Haotian Hu", Qiang Zhao", Xiaoxuan Liu, Tao Yan \\ Department of Neurology, Tianjin Medical University General Hospital, Tianjin Neurological Institute, Key Laboratory of Post-Neurotrauma, \\ Neurorepair, and Regeneration in Central Nervous System, Ministry of Education and Tianjin City, Tianjin 300052, China \\ Contributions: (I) Conception and design: H Hu, Q Zhao; (II) Administrative support: T Yan; (III) Provision of study materials or patients: H Hu; (IV) \\ Collection and assembly of data: H Hu, Q Zhao, X Liu; (V) Data analysis and interpretation: H Hu, Q Zhao; (VI) Manuscript writing: All authors; (VII) \\ Final approval of manuscript: All authors. \\ "These authors contributed equally to this work. \\ Correspondence to: Tao Yan. Department of Neurology, Tianjin Medical University General Hospital,154 Anshan Road, Heping District, Tianjin \\ 300052, China. Email: taoyan@tmu.edu.cn.
}

Background: Traumatic brain injury (TBI) evokes neurological deficits and induces cardiac dysfunction. Treatment with human umbilical cord blood cells (HUCBCs) represents a potential therapeutic strategy for TBI-induced neurological deficits. The present study aimed to determine whether HUCBCs could ameliorate the cardiac dysfunction and neurological deficits induced by TBI.

Methods: Adult male C57BL/6J mice were subjected to controlled cortical impact (CCI)-induced TBI and were treated with either HUCBCs $\left(1 \times 10^{6}\right)$ or phosphate-buffered saline (PBS), via tail vein injections, 3 days after TBI. Neurological and cognitive functions were subsequently evaluated at multiple time points after TBI and cardiac function was assessed by echocardiography 3 and 30 days after TBI. Brain and heart tissues were paraffin-embedded 30 days after TBI. Hematoxylin and eosin (H\&E) staining was performed on brain tissue sections to calculate the brain damage volume, and Picro Sirius Red (PSR) staining was performed on heart tissue sections to evaluate myocardial fibrosis. Terminal deoxynucleotide transferase dUTP nick end labeling (TUNEL) staining was employed to assess cell apoptosis 30 days after TBI. Transforming growth factor-beta (TGF- $\beta$ ) and NADPH oxidase-2 (NOX2) levels were assessed to evaluate inflammation and oxidative stress levels 30 days after TBI.

Results: TBI elicited acute and chronic cardiac deficits, identified by decreased left ventricular ejection fraction (LVEF) and fractional shortening (LVFS) values 3 and 30 days after TBI, in addition to neurological and cognitive deficits. TBI mice treated with HUCBCs exhibited enhanced LVEF and FS values 30 days after TBI compared with untreated TBI controls. HUCBC treatment significantly improved neurological and cognitive functions and reduced cardiomyocyte apoptosis, inflammatory response, oxidative stress, and cardiac fibrosis in heart tissues 30 days after TBI.

Conclusions: TBI induced both neurological deficits and cardiac dysfunction in mice, which were ameliorated by HUCBC treatment. The anti-inflammatory activities of HUCBCs may contribute to these observed therapeutic effects.

Keywords: Traumatic brain injury (TBI); human umbilical cord blood cells (HUCBCs); cardiac dysfunction; neurological deficit

Submitted Dec 03, 2019. Accepted for publication Feb 21, 2020.

doi: $10.21037 /$ atm.2020.03.52

View this article at: http://dx.doi.org/10.21037/atm.2020.03.52 


\section{Introduction}

Annually, traumatic brain injury (TBI) affects approximately 54-60 million people worldwide (1). The TBI-induced primary insult can evoke a brain parenchyma injury and triggers a secondary cascade of neurometabolic events, including cerebral edema, inflammation, and oxidative stress (2), which leads to the high incidence of mortality and disability. In addition to neurological deficits, emerging evidence has demonstrated that TBI can cause cardiac complications in mammals (3-5). Our previous studies have identified TBI-induced cardiac dysfunction, as demonstrated by significantly decreased left ventricular ejection fraction (LVEF) and fractional shortening (LVFS) values in TBI model mice (3). During epidemiological studies, patients with TBI have been reported to display cardiac deficits, including regional wall motion abnormalities, reduced LVEF values, tachycardia, and prolonged QTc, as measured using echocardiography and electrocardiograms $(4,6)$. Moreover, cardiac dysfunction after isolated TBI has been implicated in increased in-hospital mortality rates (4). Because of the increase in post-TBI morbidity and mortality rates due to TBI-induced cardiac dysfunction, new therapeutic approaches must be developed that are specifically designed not only to reduce neurological deficits but also to address the cardiac dysfunction induced by TBI.

Human umbilical cord blood cells (HUCBCs) represent an effective therapeutic approach for the treatment of various diseases, including stroke, brain injury, and myocardial infarction (MI) (7-9). HUCBCs, which are primarily composed of hematopoietic cells, mesenchymal progenitor cells, neural stem/progenitor cells, and endothelial cell precursors, have very low risks of inducing graft-versus-host disease and are easily obtainable without ethical concerns (9). HUCBCs produce neurotrophins and angiogenic growth factors, as well as microRNAs contained within exosomes, which can mediate cell- therapy-derived cardio- and neuro-restorative effects (10). Previous studies have found that HUCBC treatment can also have antiinflammatory effects and can promote functional recovery after ischemic stroke (8,11), TBI (10), and MI (12). However, the restorative effects of HUCBCs on TBIinduced cardiac dysfunction have not been investigated. In this study, we investigated the therapeutic effects and the underlying mechanisms associated with HUCBC treatment in mice with TBI-induced cardiac dysfunction and neurological deficits.

\section{Methods}

\section{Animals}

Male C57BL/6J mice (8-10 weeks) were purchased from the Vital River Corporation (Beijing, China) and were housed in the animal care facility at Tianjin Medical University General Hospital (Tianjin, China), with food and water available ad libitum and maintained on a 12-hour light/dark schedule. Experimental protocols were approved by the Tianjin Medical University General Hospital Animal Care and Use Committee. Appropriate measures were taken to ensure minimal pain and discomfort in animals.

\section{TBI induction}

The mice were anesthetized with 5\% chloral hydrate (Solarbio Life Sciences) ( $7 \mathrm{mg} / \mathrm{kg}$ ) by intraperitoneal injection. The controlled cortical impact (CCI)-induced TBI model was employed, as previously described (3). Briefly, the animal was mounted on a stereotaxic system to provide accurate coordinates for the CCI injury. The surgical site was prepared by limited shaving and disinfection, and then a small skin incision was made to expose the skull. Using a micro-drill with a trephine tip diameter of $5 \mathrm{~mm}$, a craniotomy was performed on the left parietal bone, centrally situated between bregma and lambda. Mice were subjected to a moderate CCI injury, at a velocity of $6 \mathrm{~m} / \mathrm{s}$ and a depth of $1.50 \mathrm{~mm}$, using an eCCI-6.3 device (USA). Sham animals underwent the same procedure as the CCI mice, without the cortical impact. Following the injury, bone wax was applied to fill the hole in the skull, the skin incision was sutured, and the mice were placed on a warm mat to maintain normal body temperature until recovery from anesthesia.

\section{Experimental groups}

The mice were randomly assigned to the following groups: (I) Sham TBI control; (II) TBI vehicle group: TBI mice treated with phosphate-buffered saline (PBS) as a vehicle control; and (III) HUCBC treatment group: TBI mice treated with HUCBCs (n=10 per group). The HUCBC (VCANBIO Life Sciences) treatment was performed by injecting $1 \times 10^{6}$ cells in $100 \mu \mathrm{L}$ PBS into the tail vein 3 days after TBI. In the report from company, the total number of nucleated cells was $9.94 \times 10^{8}$, and the number of CD $34^{+}$ 
cells was $32.10 \times 10^{5}$ and the ratio of $\mathrm{CD} 34^{+}$cells is $0.34 \%$ after rewarming. Cardiac function was evaluated 3 days after TBI (before treatment) and 30 days after TBI. Mice were sacrificed 30 days after TBI, and tissues were collected for immunostaining and pathological staining.

\section{Neurological and cognitive functional evaluations}

To assess motor and sensory function, a battery of tests, including the modified Neurological Severity Score (mNSS) and the foot-fault test, were performed prior to TBI and on days 1, 7, and 14 after TBI (13). The Morris Water Maze (MWM) test was performed from day 21 to 25 after TBI, to evaluate spatial and visual learning and memory function. All cognitive evaluations were performed by a researcher who was blinded to the experimental groups (14).

\section{Echocardiographic measurements}

To measure cardiac physiological functions, mice were first shaved to expose the left chest cavity and then anesthetized with isoflurane $(3.5 \%$ induction and $1-1.5 \%$ maintenance), until steady breathing was observed and the rear foot reflexes faded. Then, the left ventricular function was recorded using a VisualSonics Vevo 770 (VisualSonics, Toronto, Ontario, CA, USA), in real-time. Using 2-dimensional (2D) M-mode analysis along the short axis, the following parameters were recorded: LVEF, LVFS, LV end-systolic dimension (LVID:s), left ventricular systolic volume (LVvol:s). All primary measurements were digitized by goal-directed, diagnostically driven software, and at least 3 series of 3 heartbeat cine loops were recorded for each animal. Echocardiography was performed by a researcher who was blinded to the experimental groups.

\section{Lesion volume measurement}

On day 30 after TBI, mice were anesthetized with $10 \%$ chloral hydrate $(3 \mathrm{mg} / \mathrm{kg})$, via intraperitoneal injection, and perfused transcardially with cold PBS. Then, the brains were removed into $4 \%$ paraformaldehyde, overnight at $4{ }^{\circ} \mathrm{C}$, for fixation. Using a mouse brain matrix, each brain, with olfactory bulbs and cerebellum removed, was divided into 7 equal coronal blocks (1-mm-thick). The blocks containing injured tissue were paraffin-embedded and cut into 7- $\mu \mathrm{m}-$ thick slices, which were stained with hematoxylin and eosin (H\&E). To measure each lesion volume, one 7- $\mu \mathrm{m}-$ thick slice from each 1-mm-thick block was traced using a microcomputer imaging device (MCID, Imaging Research). The data were presented as a percentage of the total lesion volume, and the formula used to calculate the percentage of each cortical lesion volume was: [(contralateral cortical volume - ipsilateral cortical volume)/(contralateral cortical volume)] $\times 100 \%(15)$.

\section{Pathological and immunohistochemical assessments}

Mice were anesthetized with $10 \%$ chloral hydrate ( $3 \mathrm{mg} / \mathrm{kg}$ ) by intraperitoneal injection, then transcardially perfused with cold $1 \mathrm{X}$ PBS. Brain and heart tissues were isolated and fixed in $4 \%$ paraformaldehyde, overnight, followed by paraffin embedding. Next, heart samples were cut into $7-\mu \mathrm{m}$ coronal sections for pathological and immunostaining. Picro Sirius Red (PSR) staining was performed to measure the interstitial collagen fraction (ICF) (16). Primary antibodies against transforming growth factor-beta (TGF- $\beta ; 1: 250$, Santa Cruz), and NADPH oxidase-2 (NOX2; 1:400, Abcam) were employed. The nuclei were counterstained with 4'-6-diamidino-2-phenylindole (DAPI; Abcam, Cambridge, MA, USA) for $5 \mathrm{~min}$. A fluorometric terminal deoxynucleotide transferase dUTP nick end labeling (TUNEL) system (Promega, Madison) was used to assess apoptotic cells in the heart sections. Three slides were selected from each specimen, and five fields of view from each slide were digitized under $20 \times$ magnification, using a light/fluorescence microscope (Olympus, Tokyo, Japan). The percent positive areas for PSR, TGF- $\beta$, and NOX2 in the fields of view were calculated using Image Pro Plus 6.0.

\section{Statistical analysis}

All data are based on multiple independent experiments and are expressed as the mean \pm SEM. For neurological scores, such as the mNSS test, the foot-fault test, and the MWM test, the data collected at each time point were analyzed by repeated-measures analysis of variance (ANOVA), followed by least significant difference Student's $t$-tests for multiple comparisons. A one-way ANOVA, followed by Tukey's multiple comparisons test, was applied for all other data. A $\mathrm{P}$ value of less than 0.05 was considered to be statistically significant. 

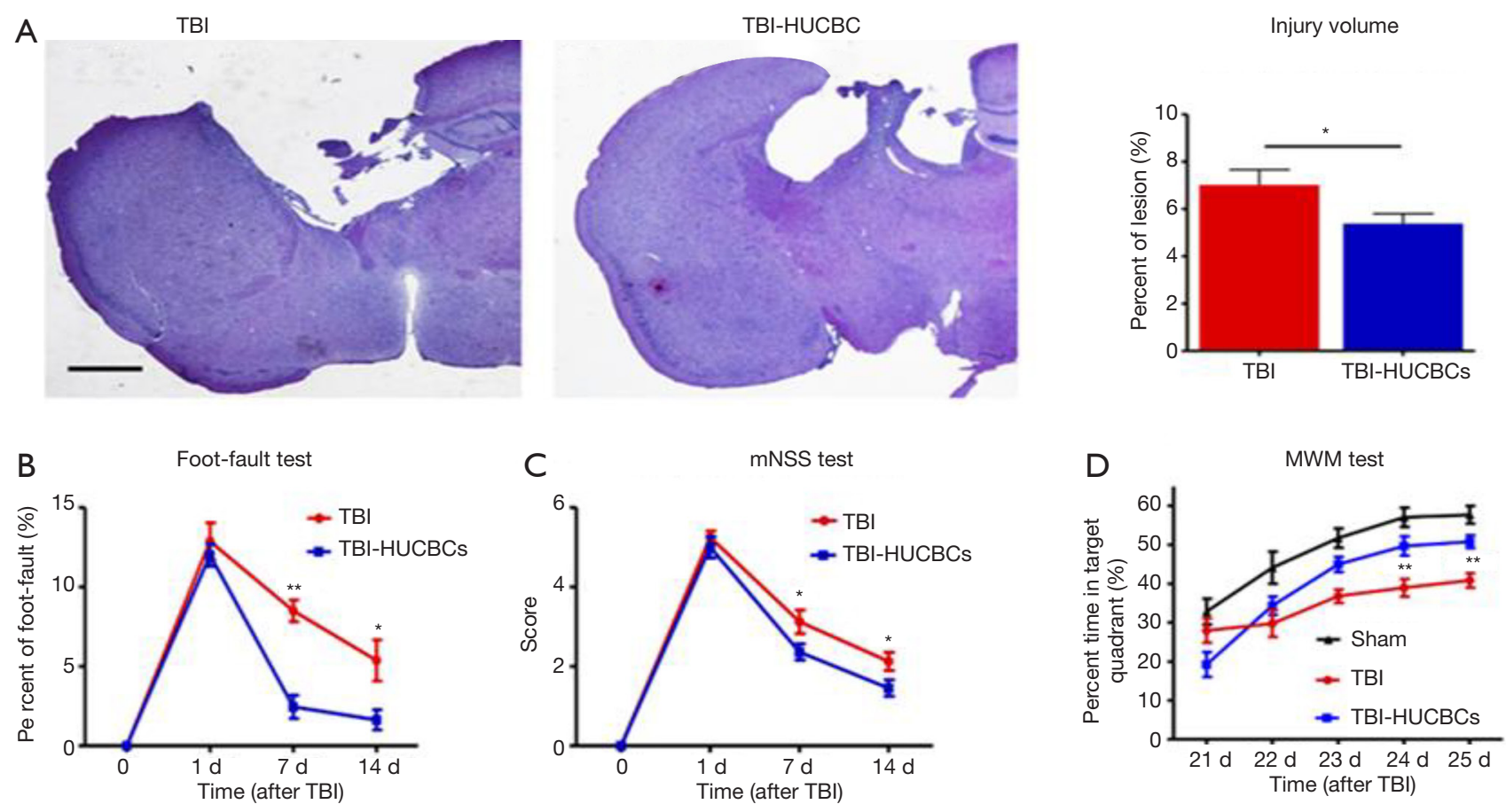

Figure 1 HUCBC treatment of TBI significantly attenuated brain lesion volume and rescued TBI-induced neurological and cognitive decline. (A) H\&E staining from brain tissue and brain lesion volume measurements. Scale bar $=1 \mathrm{~mm}$. Data are presented as the mean \pm SEM. *, $\mathrm{P}<0.05$ compared to TBI. $\mathrm{n}=10$ /group for H\&E stain; (B) foot-fault test; (C) modified neurological severity score (mNSS) test; (D) percent time spent in the target quadrant during an MWM test. Data are presented as the mean \pm SEM. *, P<0.05 TBI-PBS $v$ s. TBIHUCBCs. n=10/group. TBI, traumatic brain injury; HUCBC, human umbilical cord blood cell; H\&E, hematoxylin and eosin; PBS, phosphate-buffered saline.

\section{Results}

\section{HUCBC treatment of TBI mice significantly attenuated brain lesion volumes and rescued TBI-induced neurological and cognitive decline}

To determine whether HUCBC treatment was able to reduce brain lesion volumes during the chronic stage of TBI, H\&E staining was performed on brain tissues 30 days after TBI. Figure $1 A$ shows that HUCBC treatment significantly decreased brain lesion volumes induced by TBI compared with the TBI-PBS control mice $(\mathrm{P}<0.05)$.

To assess the efficacy of HUCBC treatment against neurological deficits after TBI, the foot-fault test and the mNSS were performed on days 1,7 , and 14 after TBI. Figure $1 B, C$ shows that $\mathrm{HUCBC}$ treatment reduced neurological deficits, as demonstrated by the decreased percentages observed for the foot-fault test and the decreased mNSS scores for HUCBC treated TBI mice compared with TBI-PBS control mice on days 7 and 14 after TBI. TBIrelated distributed volume loss is associated with a range of neuropsychological deficits, including attention, memory, and executive function defects, which have been attributed to diffuse axonal injury in animal models $(3,17)$. To determine whether HUCBC treatment can ameliorate memory deficits, the MWM test was performed from day 21 to 25 after TBI in this study. We observed that HUCBC treatment prevented the reduced percentage of target quadrant time induced by TBI (Figure 1D). Taken together, these data indicated that HUCBC treatment significantly decreased brain lesion volume and improved TBI-induced neurological and cognitive functional deficits.

\section{HUCBC treatment prevented TBI-induced cardiac dysfunction}

To test whether HUCBC treatment could improve cardiac dysfunction after TBI, echocardiography was performed 3 days after TBI (before treatment) and 30 days after TBI. Consistent with the findings of our previous study (3), we found that isolated TBI elicited cardiac dysfunction at 
3 days, resulting in reduced LVEF and LVFS values, and increased LVID:s and LVvol:s values (Figure 2A,B,C,D,E). No differences in cardiac function 3 days after TBI (before treatment) were observed between the TBI-PBS and TBIHUCBC groups (Figure 2B,C,D,E). Next, we treated TBI mice with HUCBCs or PBS, via tail vein, on the same day that cardiac testing was performed. Echocardiography was performed to assessed long-term cardiac function on day 30 after TBI. The HUCBC treatment of TBI mice markedly improved cardiac function, as assessed by the increased LVEF and LVFS values and decreased LVvol:s and LVID:s compared with those in TBI-PBS control mice (Figure 2F,G,H,I,7). Taken together, our data showed that TBI caused cardiac dysfunction, whereas HUCBC treatment attenuated TBI-induced cardiac dysfunction.

\section{HUCBC-treated TBI mice exhibited significantly decreased cardiac fibrosis and apoptosis}

A large body of evidence has demonstrated that cardiac fibrosis and apoptosis can exacerbate cardiac dysfunction in several types of heart diseases $(18,19)$. We found that TBI caused cardiac fibrosis and cardiac apoptosis during the chronic phase of TBI (3). To test whether HUCBC treatment of TBI mice could regulate cardiac fibrosis and apoptosis, PSR and TUNEL staining were performed on cardiac tissues 30 days after TBI. Figure $3 A, B$ shows that HUCBC treatment reversed TBI-induced cardiac fibrosis (Figure 3A) and apoptosis (Figure 3B) in TBI mice compared with TBI-PBS control mice.

\section{HUCBC treatment reduced the expression of inflammation factors}

Our previous studies have found that TBI-induced inflammatory responses and oxidative stress in the heart played adverse roles in TBI-induced cardiac deficits (3). To determine whether HUCBC treatment could arrest the inflammatory responses and oxidative stress observed in heart tissue after TBI, TGF- $\beta$ and NOX2 immunostaining were employed. The HUCBC treatment of TBI mice resulted in the significantly reduced expression of TGF- $\beta$ (Figure $4 A$ ) in heart tissue compared with TBI-PBS control mice. The production of NOX2 in the heart was also lower in the HUCBC-treated group than in the PBS-treated group (Figure 4B). Together, our data demonstrated that HUCBC treatment reduced the production of inflammatory factors that may contribute to TBI-induced cardiac dysfunction.

\section{Discussion}

In this study, we demonstrated, for the first time, that the HUCBC treatment of TBI mice not only decreased brain lesion volumes and reduced neurological and cognitive deficits but also improved TBI-induced cardiac deficits and decreased cardiac fibrosis, cardiac apoptosis, and the expression of inflammatory factors in heart tissue compared with PBS-treated TBI controls.

\section{HUCBC treatment ameliorated neurological and cognitive deficits induced by TBI}

Currently, neuroprotection represents a crucial strategy for the treatment of TBI. However, to date, no effective neuroprotective drugs have been identified by TBI clinical trials (20). Experimental animal studies have illustrated that neurorestorative approaches, which boost angiogenesis (21), axonal remodeling, neurogenesis, and synaptogenesis, represent promising opportunities for the treatment of TBI (20). Cell-based therapy is a vital neurorestorative strategy and adipose-derived stem cells (ADSC), amniotic fluid stem cells (AFSC), bone marrow stromal cells (BMSC), and the mononuclear fraction of human umbilical cord blood (HUCB) have all displayed neuroprotective properties in TBI models, via reductions in inflammatory responses, neuronal death, and the rescue of neurological and cognitive functions (22-26). However, whether HUCBC treatment represents an effective approach for TBI-induced neurological deficits has not been wellinvestigated. Here, our data demonstrated that HUCBC treatment restored TBI-induced neurological deficits in mice, accompanied by decreased brain injury volumes. In addition, our data indicated that HUCBC treatment improved cognitive decline after TBI. In several stroke studies, HUCBC treatments have been reported to enhance Angiopoietin-1 expression, downregulate the receptor for advanced glycation end products (RAGE) (27), remodel white matter and vascular tissue, and accelerate M2 macrophage polarization to achieve neuroprotective effects $(11,28)$, which may explain the neuroprotective effects observed in this study and should be explored more thoroughly in the future.

\section{HUCBC treatment rescued cardiac deficits and corresponding heart pathological injuries}

Brain-heart crosstalk after brain injury has become an 

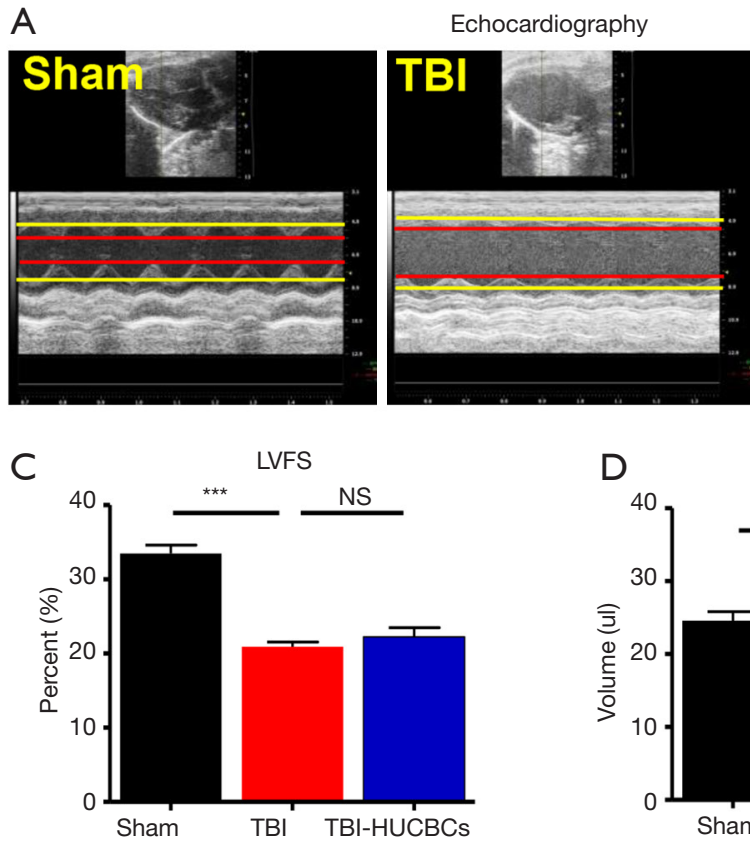
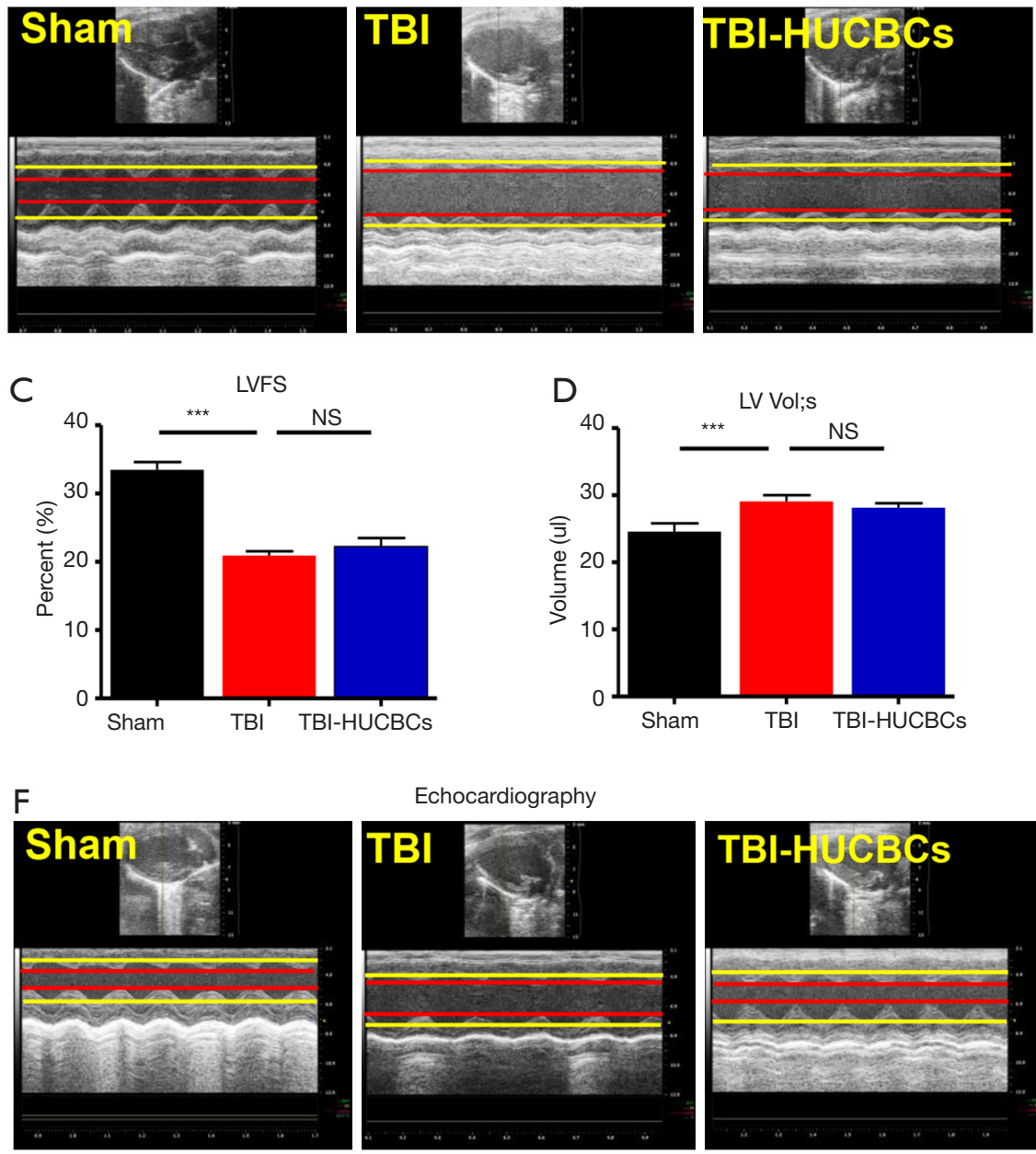

Echocardiography
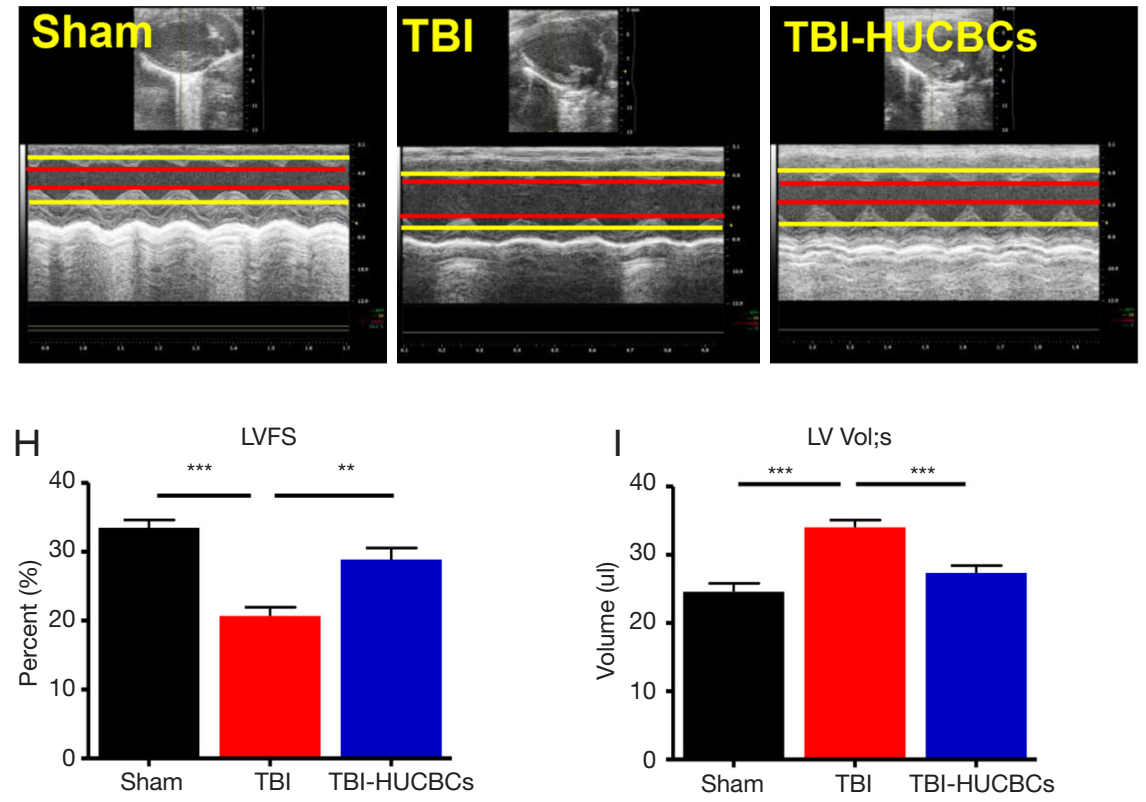

B LVEF

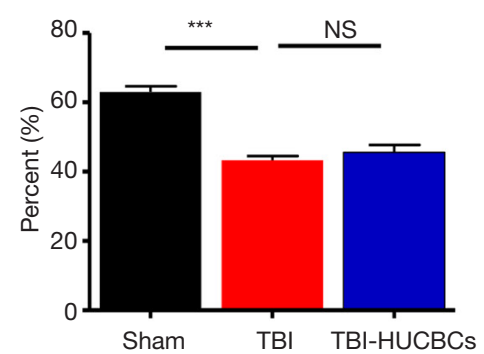

$\mathrm{E}$
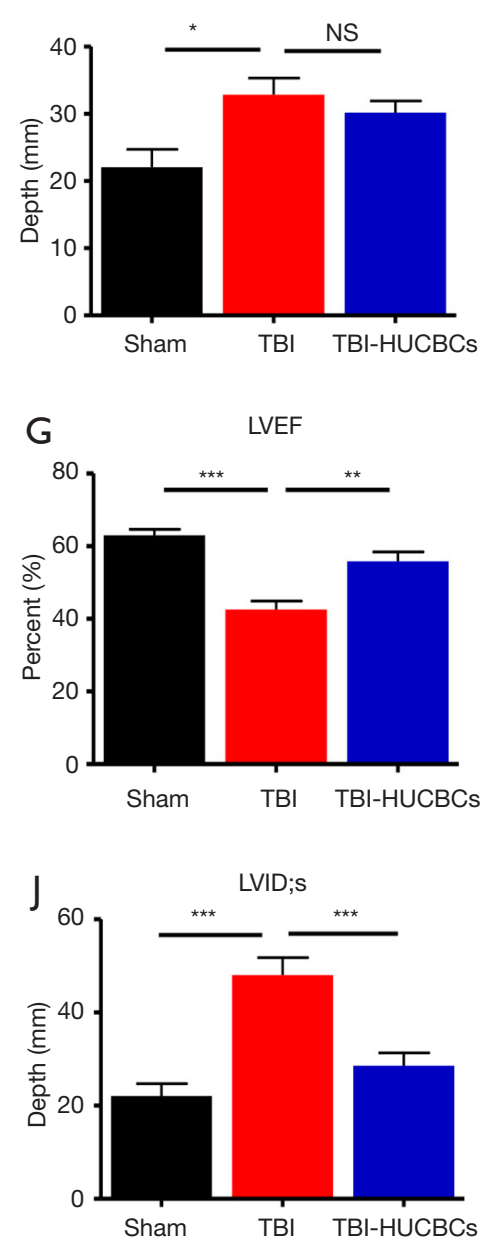

Figure 2 TBI mice treated with HUCBCs exhibited significantly improved cardiac dysfunction compared with TBI mice treated with PBS. (A) Representative echocardiography pictures, 3 days after TBI; (B) LVEF, 3 days after TBI; (C) LVFS, 3 days after TBI; (D) LV Vol;s, 3 days after TBI; (E) LVID;s, 3 days after TBI; (F) representative echocardiography pictures, 30 days after TBI; (G) LVEF, 30 days after TBI; (H) LVFS, 30 days after TBI; (I) LV Vol;s, 30 days after TBI; (J) LVID;s. 30 days after TBI. Data are presented as the mean \pm SEM. *, $\mathrm{P}<0.05,{ }^{* *}, \mathrm{P}<0.01$, and ${ }^{* * *}, \mathrm{P}<0.001$ compared to sham or compared to TBI, either 3 or 30 days after TBI. NS, not significant; $\mathrm{n}=10$ /group. TBI, traumatic brain injury; HUCBC, human umbilical cord blood cell; PBS, phosphate-buffered saline; LVEF, left ventricular ejection fraction; LVFS, left ventricular fractional shortening. 

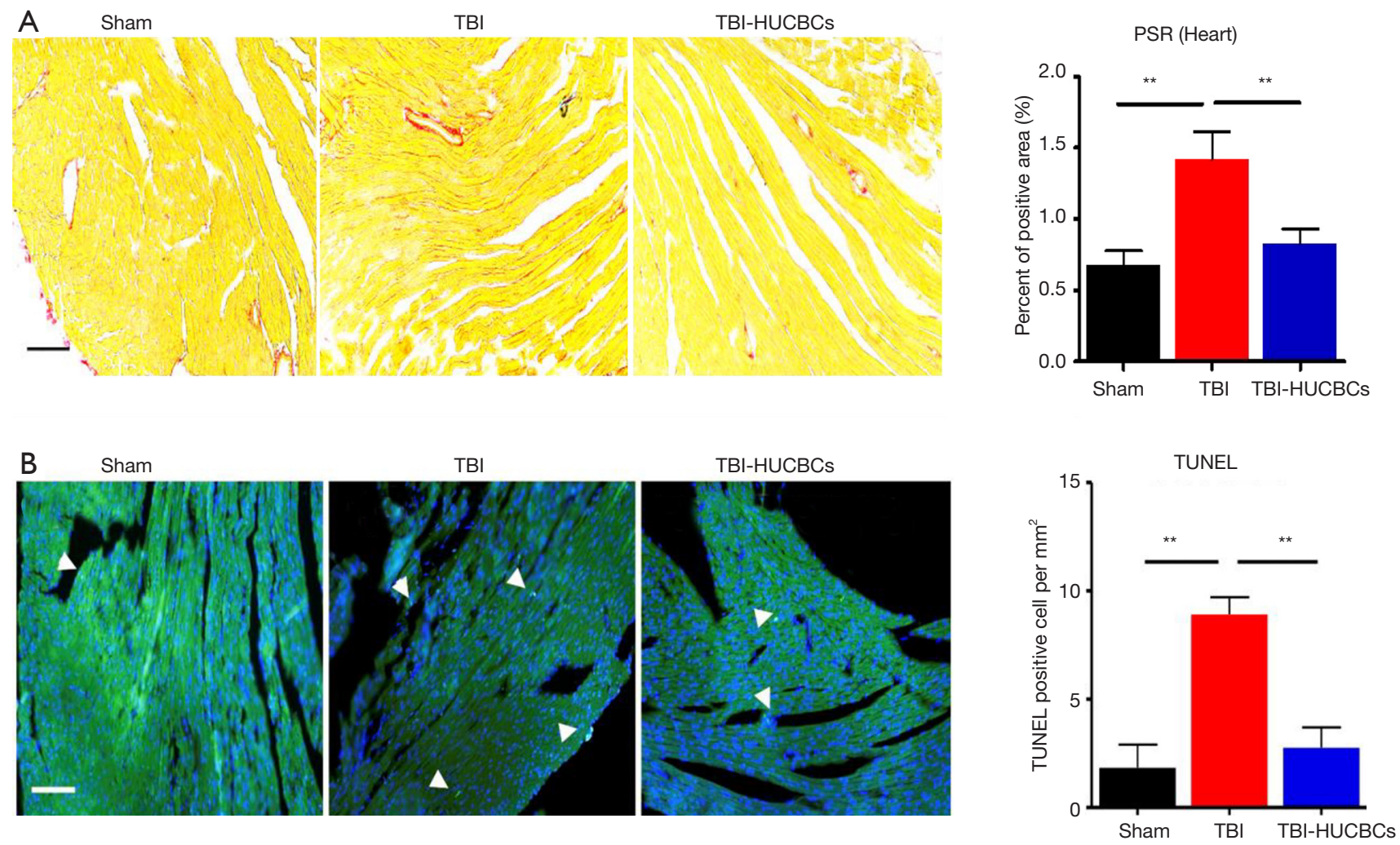

Figure 3 TBI mice treated with HUCBCs exhibited significantly decreased cardiac fibrosis and apoptosis. (A) PSR staining and interstitial collagen measurements performed 30 days after TBI; (B) TUNEL staining and quantitative data collected 30 days after TBI. Scale bar in $\mathrm{A}-\mathrm{B}=100 \mu \mathrm{m}$. Data are presented as the mean \pm SEM. *, $\mathrm{P}<0.05$ and ${ }^{* *}, \mathrm{P}<0.05$ compared to sham or compared to TBI 30 days after TBI; n=10/group. TBI, traumatic brain injury; HUCBC, human umbilical cord blood cell; PSR, Picro Sirius Red.

emerging research hotspot (29). To date, a considerable number of clinical and experimental animal studies have shown that acute and chronic cardiac functional damage occurs after stroke and TBI, which can influence neurological outcomes and increase the burden of everyday living in TBI patients (3,30-32). Recently, several studies have employed HUCBCs to treat MI in mice, which profoundly reduced the MI-induced infarct size and improved MI-induced cardiac dysfunction, accompanied by a weakened inflammatory response $(7,12)$. Interestingly, our data demonstrated that HUCBC treatment prevented the progression of cardiac deficits by increasing LVEF and FS values and decreasing $L V v o l: s$ and LVvol:d values, as well as reducing cardiac fibrosis and apoptosis compared with TBIPBS control mice. Therefore, the HUCBC treatment of TBI mice not only resulted in brain plasticity benefits but also significantly decreased cardiac deficits.

\section{The potential mechanism through which HUCBC treatment ameliorated TBI-induced cardiac deficits}

Finally, we attempted to elucidate the potential mechanisms through which HUCBC treatments ameliorated TBIinduced cardiac deficits. Our previously published data have demonstrated that the immune response mediated cardiac lesions after TBI (3). We observed that TBI resulted in the overexpression of TGF- $\beta$ and NOX2 in heart tissues. Other evidence has demonstrated that TGF- $\beta$ plays a crucial role in the pathogenesis of cardiac remodeling and fibrosis, leading to cardiac stiffness and systolic and diastolic dysfunction, which manifests as reduced LVEF and FS values, by regulating the differentiation and collagen secretion of cardiac fibroblasts (33). Moreover, a great deal of evidence has revealed significantly increased TGF- $\beta$ expression in the heart during MI, heart failure, 

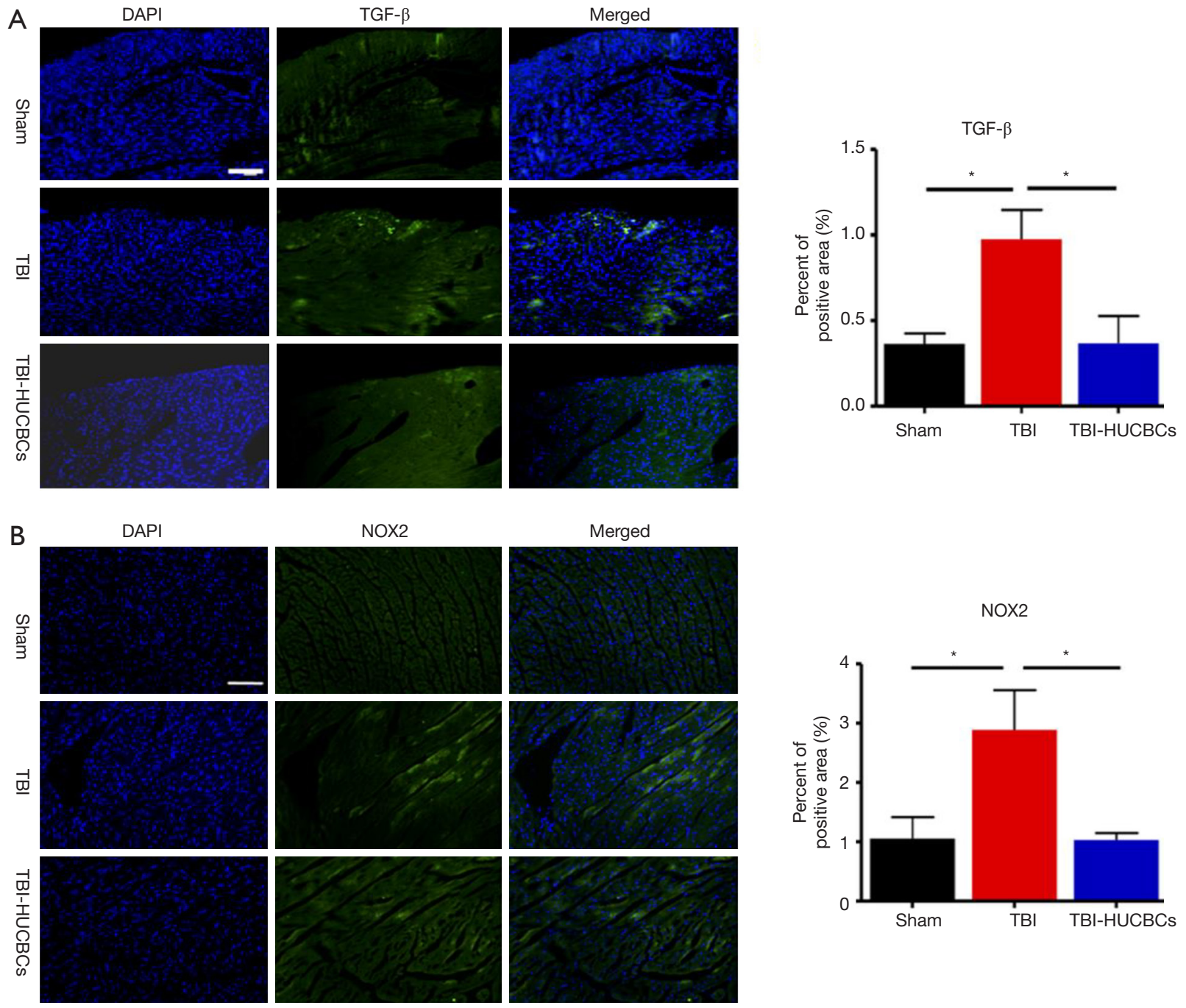

Figure 4 TBI mice treated with HUCBCs showed reduced expression levels of inflammatory factors. (A) TGF- $\beta$ staining and quantitative data, 30 days after TBI; (B) NOX2 staining and quantitative data, 30 days after TBI. Scale bars in A-B =100 $\mu \mathrm{m}$. Data are presented as the mean \pm SEM. *, $\mathrm{P}<0.05$ compared to sham or compared to TBI; $\mathrm{n}=10$ /group. TBI, traumatic brain injury; HUCBC, human umbilical cord blood cell.

dilated and hypertrophic cardiomyopathy (34-36). NOX2, which is primarily expressed in cardiac endothelial cells, fibroblasts, and leukocytes (37), may be detrimental during atherosclerosis and hypertension (38). NOX2 can boost the transition from fibroblasts to myofibroblasts and mediate cardiac inflammation, fibrosis, and heart hypertrophy (39). In a study examining MI, HUCBC treatment following acute MIs was shown to prevent inflammatory cytokine overexpression in the infarcted myocardium (12). Moreover, studies have demonstrated that HUCBC treatments can reduce the expression of inflammation factors during other diseases $(40,41)$. Consistently, in current study, we observed that the HUCBC treatment of TBI mice resulted in anti-inflammatory effects, including decreased TGF- $\beta$ and NOX2 expression levels in heart tissue, which may contribute to HUCBCs-mediated improvements in cardiac deficits.

\section{Limitation}

This study focuses on the effects of HUCBC treatments on TBI-induced cardiac dysfunction. However, the 
mechanisms through which HUCBC treatment mediated beneficial brain-heart interaction effects remain unclear and are worth exploring further.

\section{Conclusions}

TBI induces both neurological deficits and cardiac dysfunction in mice. HUCBC treatment ameliorated TBIinduced neurological and cardiac dysfunction in mice. HUCBC treatment may represent a potential therapeutic approach for TBI-induced deficits in both brain and heart.

\section{Acknowledgments}

Funding: This work was supported by the National Natural Science Foundation of China, Grant Numbers: 81671144, 91746205; Tianjin Natural Science Foundation Grant, Grant/Award Number: 17JCZDJC36100.

\section{Footnote}

Conflicts of Interest: The authors have no conflicts of interest to declare

Ethical Statement: The authors are accountable for all aspects of the work in ensuring that questions related to the accuracy or integrity of any part of the work are appropriately investigated and resolved. Experimental protocols were approved by the Tianjin Medical University General Hospital Animal Care and Use Committee.

Open Access Statement: This is an Open Access article distributed in accordance with the Creative Commons Attribution-NonCommercial-NoDerivs 4.0 International License (CC BY-NC-ND 4.0), which permits the noncommercial replication and distribution of the article with the strict proviso that no changes or edits are made and the original work is properly cited (including links to both the formal publication through the relevant DOI and the license). See: https://creativecommons.org/licenses/by-nc-nd/4.0/.

\section{References}

1. Feigin VL, Theadom A, Barker-Collo S, et al. Incidence of traumatic brain injury in New Zealand: a population-based study. Lancet Neurol 2013;12:53-64.

2. Maas AIR, Menon DK, Adelson PD, et al. Traumatic brain injury: integrated approaches to improve prevention, clinical care, and research. Lancet Neurol 2017;16:987-1048.

3. Zhao Q, Yan T, Li L, et al. Immune Response Mediates Cardiac Dysfunction after Traumatic Brain Injury. J Neurotrauma 2019;36:619-29.

4. Prathep S, Sharma D, Hallman M, et al. Preliminary report on cardiac dysfunction after isolated traumatic brain injury. Crit Care Med 2014;42:142-7.

5. Ozisik K, Yildirim E, Kaplan S, et al. Ultrastructural changes of rat cardiac myocytes in a time-dependent manner after traumatic brain injury. Am J Transplant 2004;4:900-4.

6. Krishnamoorthy V, Prathep S, Sharma D, et al. Association between electrocardiographic findings and cardiac dysfunction in adult isolated traumatic brain injury. Indian J Crit Care Med 2014;18:570-4.

7. Henning RJ, Burgos JD, Vasko M, et al. Human cord blood cells and myocardial infarction: effect of dose and route of administration on infarct size. Cell Transplant 2007;16:907-17.

8. Chen J, Sanberg PR, Li Y, et al. Intravenous administration of human umbilical cord blood reduces behavioral deficits after stroke in rats. Stroke 2001;32:2682-8.

9. Venkat P, Chopp M, Chen J. Cell-Based and Exosome Therapy in Diabetic Stroke. Stem Cells Transl Med 2018;7:451-5.

10. Zhao J, Chen N, Shen N, et al. Transplantation of human umbilical cord blood mesenchymal stem cells to treat a rat model of traumatic brain injury. Neural Regen Res 2012;7:741-8.

11. Yan $T$, Venkat $P, Y e X$, et al. HUCBCs increase angiopoietin 1 and induce neurorestorative effects after stroke in T1DM rats. CNS Neurosci Ther 2014;20:935-44.

12. Henning RJ, Shariff M, Eadula U, et al. Human cord blood mononuclear cells decrease cytokines and inflammatory cells in acute myocardial infarction. Stem Cells Dev 2008;17:1207-19.

13. Cui X, Chopp M, Zacharek A, et al. D-4F Decreases White Matter Damage After Stroke in Mice. Stroke 2016;47:214-20.

14. Venkat P, Chopp M, Zacharek A, et al. White matter damage and glymphatic dysfunction in a model of vascular dementia in rats with no prior vascular pathologies. Neurobiol Aging 2017;50:96-106.

15. Swanson RA, Morton MT, Tsao-Wu G, et al. A semiautomated method for measuring brain infarct volume. J Cereb Blood Flow Metab 1990;10:290-3.

16. Mito S, Ozono R, Oshima T, et al. Myocardial 
protection against pressure overload in mice lacking Bach1, a transcriptional repressor of heme oxygenase-1. Hypertension 2008;51:1570-7.

17. Liraz-Zaltsman S, Alexandrovich AG, Trembovler V, et al. Regional sensitivity to neuroinflammation: in vivo and in vitro studies. Synapse 2011;65:634-42.

18. Tanai E, Frantz S. Pathophysiology of Heart Failure. Compr Physiol 2015;6:187-214.

19. Sabbah HN, Sharov VG, Lesch M, et al. Progression of heart failure: a role for interstitial fibrosis. Mol Cell Biochem 1995;147:29-34.

20. Xiong Y, Mahmood A, Chopp M. Neurorestorative treatments for traumatic brain injury. Discov Med 2010;10:434-42.

21. Wang Z, Zheng L, Lian C, et al. Umbilical Cord-Derived Mesenchymal Stem Cells Relieve Hind limb Ischemia by Promoting Angiogenesis in Mice. Stem Cells Dev 2019;28:1384-97.

22. Gincberg G, Shohami E, Trembovler V, et al. Nerve growth factor plays a role in the neurotherapeutic effect of a CD45(+) pan-hematopoietic subpopulation derived from human umbilical cord blood in a traumatic brain injury model. Cytotherapy 2018;20:245-61.

23. Mahmood A, Lu D, Qu C, et al. Long-term recovery after bone marrow stromal cell treatment of traumatic brain injury in rats. J Neurosurg 2006;104:272-7.

24. Tajiri N, Acosta S, Glover LE, et al. Intravenous grafts of amniotic fluid-derived stem cells induce endogenous cell proliferation and attenuate behavioral deficits in ischemic stroke rats. PLoS One 2012;7:e43779.

25. Kim HJ, Lee JH, Kim SH. Therapeutic effects of human mesenchymal stem cells on traumatic brain injury in rats: secretion of neurotrophic factors and inhibition of apoptosis. J Neurotrauma 2010;27:131-8.

26. Maegele M, Schaefer U. Stem cell-based cellular replacement strategies following traumatic brain injury (TBI). Minim Invasive Ther Allied Technol 2008;17:119-31.

27. Son M, Oh S, Park H, et al. Protection against RAGEmediated neuronal cell death by sRAGE-secreting human mesenchymal stem cells in 5xFAD transgenic mouse model. Brain Behav Immun 2017;66:347-58.

28. Yan T, Venkat P, Chopp M, et al. Neurorestorative Therapy of Stroke in Type 2 Diabetes Mellitus Rats Treated With Human Umbilical Cord Blood Cells. Stroke 2015;46:2599-606.

29. Chen Z, Venkat P, Seyfried D, et al. Brain-Heart Interaction: Cardiac Complications After Stroke. Circ Res
2017;121:451-68.

30. Greenhoot JH, Reichenbach DD. Cardiac injury and subarachnoid hemorrhage. A clinical, pathological, and physiological correlation. J Neurosurg 1969;30:521-31.

31. Chen J, Cui C, Yang X, et al. MiR-126 Affects Brain-Heart Interaction after Cerebral Ischemic Stroke. Transl Stroke Res 2017;8:374-85.

32. Finsterer J, Wahbi K. CNS-disease affecting the heart: brain-heart disorders. J Neurol Sci 2014;345:8-14.

33. Dobaczewski $M$, Chen $W$, Frangogiannis NG. Transforming growth factor (TGF)-beta signaling in cardiac remodeling. J Mol Cell Cardiol 2011;51:600-6.

34. Rosenkranz S, Flesch M, Amann K, et al. Alterations of beta-adrenergic signaling and cardiac hypertrophy in transgenic mice overexpressing TGF-beta(1). Am J Physiol Heart Circ Physiol 2002;283:H1253-62.

35. Yan T, Chen Z, Chopp M, et al. Inflammatory responses mediate brain-heart interaction after ischemic stroke in adult mice. J Cereb Blood Flow Metab 2018. [Epub ahead of print].

36. Liu G, Ma C, Yang H, et al. Transforming growth factor beta and its role in heart disease. Exp Ther Med 2017;13:2123-8.

37. Braunersreuther V, Montecucco F, Asrih M, et al. Role of NADPH oxidase isoforms NOX1, NOX2 and NOX4 in myocardial ischemia/reperfusion injury. J Mol Cell Cardiol 2013;64:99-107.

38. Sirker A, Zhang M, Shah AM. NADPH oxidases in cardiovascular disease: insights from in vivo models and clinical studies. Basic Res Cardiol 2011;106:735-47.

39. Liu Y, Zhang J. Nox2 contributes to cardiac fibrosis in diabetic cardiomyopathy in a transforming growth factor-beta dependent manner. Int J Clin Exp Pathol 2015;8:10908-14.

40. Vendrame M, Gemma C, de Mesquita D, et al. Antiinflammatory effects of human cord blood cells in a rat model of stroke. Stem Cells Dev 2005;14:595-604.

41. Chen J, Ning R, Zacharek A, et al. MiR-126 Contributes to Human Umbilical Cord Blood Cell-Induced Neurorestorative Effects After Stroke in Type-2 Diabetic Mice. Stem Cells 2016;34:102-13.

Cite this article as: $\mathrm{Hu} \mathrm{H}$, Zhao Q, Liu X, Yan T. Human umbilical cord blood cells rescued traumatic brain injuryinduced cardiac and neurological deficits. Ann Transl Med 2020;8(6):278. doi: $10.21037 /$ atm.2020.03.52 